\title{
Long-range temporal correlations in the brain distinguish conscious wakefulness from induced unconsciousness
}

\author{
Thomas Thiery $^{\mathrm{a}, *}$, Tarek Lajnef ${ }^{\mathrm{a}}$, Etienne Combrisson ${ }^{\mathrm{a}, \mathrm{b}, \mathrm{c}}$, Arthur Dehgan ${ }^{\mathrm{a}}$, Pierre Rainville ${ }^{\mathrm{d}}$, \\ George A. Mashour ${ }^{\mathrm{e}}$, Stefanie Blain-Moraes ${ }^{\mathrm{f}}$, Karim Jerbi ${ }^{\mathrm{a}}$ \\ ${ }^{a}$ Psychology Department, University of Montreal, QC, Canada \\ ${ }^{\mathrm{b}}$ Center of Research and Innovation in Sport, Mental Processes and Motor Performance, University Claude Bernard Lyon I, University of Lyon, Villeurbanne, France \\ ${ }^{c}$ Brain Dynamics and Cognition, Lyon Neuroscience Research Center, INSERM U1028, UMR 5292, University of Lyon, Villeurbanne, France \\ ${ }^{\mathrm{d}}$ Department of Stomatology, University of Montreal, QC, Canada \\ ${ }^{\mathrm{e}}$ Center for Consciousness Science, Department of Anesthesiology, University of Michigan, USA \\ ${ }^{\mathrm{f}}$ School of Physical and Occupational Therapy, McGill University, Montreal, QC, Canada
}

\section{A R T I C L E I N F O}

\section{Keywords:}

Consciousness

Unconsciousness

EEG

Oscillations

Detrended fluctuation analysis (DFA)

Alpha oscillations

Anesthesia

\begin{abstract}
A B S T R A C T
Rhythmic neuronal synchronization across large-scale networks is thought to play a key role in the regulation of conscious states. Changes in neuronal oscillation amplitude across states of consciousness have been widely reported, but little is known about possible changes in the temporal dynamics of these oscillations. The temporal structure of brain oscillations may provide novel insights into the neural mechanisms underlying consciousness. To address this question, we examined long-range temporal correlations (LRTC) of EEG oscillation amplitudes recorded during both wakefulness and anesthetic-induced unconsciousness. Importantly, the time-varying EEG oscillation envelopes were assessed over the course of a sevoflurane sedation protocol during which the participants alternated between states of consciousness and unconsciousness. Both spectral power and LRTC in oscillation amplitude were computed across multiple frequency bands. State-dependent differences in these features were assessed using non-parametric tests and supervised machine learning. We found that periods of unconsciousness were associated with increases in LRTC in beta (15-30Hz) amplitude over frontocentral channels and with a suppression of alpha $(8-13 \mathrm{~Hz})$ amplitude over occipitoparietal electrodes. Moreover, classifiers trained to predict states of consciousness on single epochs demonstrated that the combination of beta LRTC with alpha amplitude provided the highest classification accuracy (above 80\%). These results suggest that loss of consciousness is accompanied by an augmentation of temporal persistence in neuronal oscillation amplitude, which may reflect an increase in regularity and a decrease in network repertoire compared to the brain's activity during resting-state consciousness.
\end{abstract}

\section{Introduction}

Anesthetics are a useful tool for the study of human consciousness, as they can be titrated to pharmacologically induce the loss of consciousness (LOC) and the recovery of consciousness (ROC) in a controlled experimental design. Anesthetic-induced LOC usually coincides with a disruption of the brain's ability to integrate information (Alkire et al., 2008). Consistent with this view, studies have shown decreased EEG complexity at the time when consciousness was lost (Lee et al., 2010; Sarasso et al., 2015; Wang et al., 2017), as well as a changes in EEG global coherence (John and Prichep, 2005; Cimenser et al., 2011; Akeju et al., 2014). Analysis of EEG during propofol- and sevoflurane-induced unconsciousness further indicates a breakdown of the spatiotemporal organization around the gamma band (Pal et al., 2016), and a decreased frontoparietal connectivity associated with propofol, sevoflurane and ketamine-induced unconsciousness (Lee et al., 2009, 2013; Boveroux et al., 2010; Jordan et al., 2013; Palanca et al., 2015; Bonhomme et al., 2016; Ranft et al., 2016). Additionally, several studies have demonstrated the importance of posterior alpha suppression and alpha anteriorization during anesthetic-induced unconsciousness (Gugino et al., 2001; Feshchenko et al., 2004; Blain-Moraes et al., 2015; Purdon et al., 2015; Pavone et al., 2017). These studies have focused on properties of local oscillations (e.g. power) and the functional connectivity between brain regions, but less is known

\footnotetext{
* Corresponding author.

E-mail address: thomas.thiery@umontreal.ca (T. Thiery).
} 
about changes in long-range temporal properties of brain oscillations associated with states of consciousness.

The brain has been characterized according to its scale-invariant properties, reflected by a $1 /$ f-like spectrum (Bak et al., 1987). This power-law scaling reflects arrhythmic brain activity with no predominant temporal scale (i.e., scale-free), and has been reported in MEG, EEG and fMRI data (Ciuciu et al., 2012; He, 2014). Some studies have used multiscale formalisms applied to the raw EEG signal, and have emphasized the relevance of scaling properties of the raw signal to predict the depth of anesthesia and measure the level of consciousness (Gifani et al., 2006; Jospin et al., 2007; Lalitha and Eswaran, 2007; Nguyen-Ky et al., 2010; Liang et al., 2015; Li et al., 2017). Interestingly, their results were consistent with bispectral index values, which are commonly used to identify anesthesia states in clinical settings. Although critical states can be detected in BOLD and raw EEG signal, they can also be observed in the amplitude-envelope of oscillations.

Beyond assessing scale-free dynamics of the raw brain signals, a growing body of literature provides evidence for the importance of investigating the scaling properties of brain signal amplitudes (i.e. envelopes) in specific frequency bands (Linkenkaer-Hansen et al., 2001). This allows us to probe their long-range temporal correlations (LRTC) and thereby provides a measure of how rhythmic neuronal activity propagates in time. Several studies have demonstrated the presence of LRTC in the amplitude dynamics of theta, alpha and beta frequency bands (Linkenkaer-Hansen et al., 2004; Nikulin and Brismar, 2004; 2005; Fedele et al., 2016) across a wide age range (Berthouze et al., 2010). Interestingly, pathological alterations of LRTC in brain oscillations were previously shown in Alzheimer's disease (Montez et al., 2009), major depressive disorder (Linkenkaer-Hansen et al., 2005; Bornas et al., 2015), schizophrenia (Slezin et al., 2007; Nikulin et al., 2012) and epilepsy patients (Monto et al., 2007). However, whether and how LRTC in specific frequency bands are modulated across states of consciousness remains unknown.

In the present study, we investigated the association of the LRTC of brain oscillations with varying states of consciousness. We hypothesized that reduced information processing during unconsciousness would be associated with increased brain signal regularity, which should translate into increased LRTC in the frequency bands most relevant for information integration. Secondly, we hypothesized that LRTC effects would not necessarily coincide with changes in mean spectral power in terms of frequency range and spatial distribution. To this end, we evaluated changes in the long-range temporal structure of band-limited EEG amplitude fluctuations (as well as standard mean spectral amplitude) during sevoflurane-induced unconsciousness in healthy participants. Importantly, this study protocol employed a gradual increase in the level of sevoflurane to bring participants to the border of conscious state transitions, leading to multiple successive switches between the state of consciousness and unconsciousness.

\section{Materials and methods}

\section{Participants}

Ten healthy volunteers were recruited and provided their written and informed consent after a careful discussion of risks and benefits to participate in this study. The study was conducted at the University of Michigan Medical School and approved by the Institutional Review Board (HUM00061087). Two volunteers withdrew during administration of sevoflurane due to discomfort and one subject's EEG data were suboptimal due to excessive motion. Therefore, data from seven healthy volunteers (four males, 20-23 yrs. old) were included in the analysis. These data were previously published in a study investigating alpha anteriorization, cross-frequency coupling, and long-range phase correlations (Blain-Moraes et al., 2015). They have been re-analyzed in this study to test a distinct hypothesis.

\section{Anesthetic protocol}

Each experiment was conducted by two trained anesthesiologists. At the beginning of sevoflurane administration, participants were instructed to squeeze an object in either their left hand or right hand while instructions were delivered every 30 seconds through an audio loop. Left and right commands were randomized. Participants kept their eyes closed during waking states throughout the experiment and received sevoflurane anesthesia by a secured face mask with an initial concentration of $0.4 \%$ in high-flow oxygen. The protocol of sevoflurane administration follows the procedure introduced by Suzuki and colleagues (Suzuki et al., 1998). Sevoflurane was administered at a given level for a 15-min equilibration period to achieve steady state, followed by $10 \mathrm{~min}$ at the target concentration. Sevoflurane concentration was increased by increments of $0.2 \%$ until loss of consciousness (LOC) was achieved. After 10 minutes of unconsciousness, the reverse protocol (equilibration followed by steady-state period in increments of $0.2 \%$ ) was employed until the participant regained consciousness. After recovery of consciousness (ROC), anesthetic concentration was still titrated downward until end-tidal values were $0 \%$. More details concerning the anesthetic protocol are available in Blain-Moraes and colleagues (Blain-Moraes et al., 2015).

\section{Behavioral state analysis}

Before sevoflurane exposure, the participants were given objects in each hand that would emit a sound when squeezed. They were instructed to squeeze the object in either their left hand or right hand when sevoflurane administration began; left/right commands were randomized and instructions were delivered every 30 seconds. Response presence or absence was observed and recorded in the data collection software by two investigators throughout the experimental period. To compare and pool data across participants, data segments were split into two categories using two types of behavioral time markers: LOC corresponded to the first failure to respond to an auditory command and ROC to the first positive response to an auditory command following LOC. We then defined in each subject the consciousness vs unconsciousness data segments based on these individual responsiveness profiles: (1) Conscious periods were defined as those directly preceding LOC or following ROC markers and (2) Unconscious periods were defined as those between LOC and ROC.

\section{EEG recordings and pre-processing}

The EEG was acquired using a 64-channel sensor net from Electrical Geodesics, Inc. (Eugene, OR) and all channels were referenced to the vertex. Electrode impedances were kept below $50 \mathrm{k} \Omega$ and EEG signals were collected at a sampling rate of $500 \mathrm{~Hz}$. EEG signals were rereferenced to an average reference and visually inspected to reject epochs and channels with noise or non-physiological artefacts. We used the individual responsiveness profiles to discriminate conscious from unconscious segments in the raw EEG data. For each subject, we ended up with two separated raw datasets, one for the conscious condition and one for the unconscious condition. We then segmented the data from both conditions in 60 second epochs with no overlap in order to match the number of samples we obtained in the detrended fluctuation analysis (DFA) (see next section). The average number of 60 second conscious epochs per participant was $68( \pm 20)$, while the average number of unconscious epochs was $34( \pm 13)$.

\section{Instantaneous amplitude of neuronal oscillations}

The instantaneous amplitude of the EEG signals was computed in five different frequency bands: delta $(1-4 \mathrm{~Hz})$, theta $(4-7 \mathrm{~Hz})$, alpha $(8-13 \mathrm{~Hz})$, beta $(15-30 \mathrm{~Hz})$ and gamma $(30-60 \mathrm{~Hz})$. This was achieved by first filtering the raw EEG signals using a finite impulse response 
filtering (FIR1, order $=3$ ) and then computing the Hilbert transform (Foster et al., 2016). Fig. 2A shows an example of the raw signal, and Fig. 2B shows the filtered signal and its instantaneous amplitude in the beta band. The amplitude was obtained for each 60-s epoch and for each channel and condition separately (conscious and unconscious). Then, the overall mean amplitude of neuronal oscillations in each frequency band was computed by averaging the amplitude values across all available data samples for each channel and condition (conscious and unconscious) separately.

\section{Detrended fluctuation analysis (DFA)}

The presence of LRTC indicates that the temporal auto-correlations attenuate very slowly in time, according to a power-law. Thus, slow attenuation of LRTC is an indication of how neuronal events develop over time. It provides information about the long-term temporal structure of the complex patterns of ongoing activity, which in turn is thought to be related to the integrity of multiple interconnected populations of neurons (e.g., Nikulin et al., 2012). In EEG recordings, DFA can be applied to detect long-range temporal correlations (auto-correlations) either in the raw time-domain signals or alternatively in the temporal dynamics of the signal envelope (amplitude) in a given frequency band (Peng et al., 1995; Kantelhardt et al., 2001). In the present study, DFA was used to analyze the decay of temporal auto-correlations in the time range of $5-50 \mathrm{~s}$. The processing of broadband EEG signal to enable quantification of LRTC using DFA has been explained in detail elsewhere (Linkenkaer-Hansen et al., 2001; Nikulin and Brismar, 2005). In brief, the DFA measures the scaling of the root-mean-square fluctuation of the integrated and linearly detrended signals, $\mathrm{F}(\mathrm{t})$, as a function of time window size $t$ (Fig. 2C). The DFA exponent is the slope of the fluctuation function shown in Fig. 2C. A DFA scaling exponent, also termed the "self-similarity parameter" (Lux and Marchesi, 1999), is extracted with linear regression in double-logarithmic coordinates using a least-squares algorithm. A scaling exponent between 0.5 and 1.0 indicates a greater persistence of temporal correlations (i.e. temporal autocorrelations), while scaling exponents between 0 and 0.5 reflect decreased signal persistence (i.e. temporal anti-correlations). An uncorrelated signal is characterized by an exponent of 0.5 . This method is thought to have several advantages over other options such as the Generalized Hurst Exponent or the autocorrelation function (Peng et al., 1995), since it requires less strict assumptions about the stationarity of the signal and provides greater accuracy in the estimates of correlations (Gao et al., 2006; Linkenkaer-Hansen et al., 2007). Previous studies have shown that electrophysiological brain signals generally have scaling exponents above 0.5 (Linkenkaer-Hansen et al., 2001, 2004; Nikulin and Brismar, 2004; 2005; Berthouze et al., 2010; Fedele et al., 2016). Here we measured LRTC in the signal on single 60-s epochs. As DFA can be affected by the length of the signal it is calculated on, we conducted an additional analysis were LRTC were computed on the whole signal. Importantly, we found similar results in both cases.

\section{Statistical analysis}

We conducted a two-sided permutation based pseudo $t$-test corrected with maximum statistics using exhaustive permutations (number of
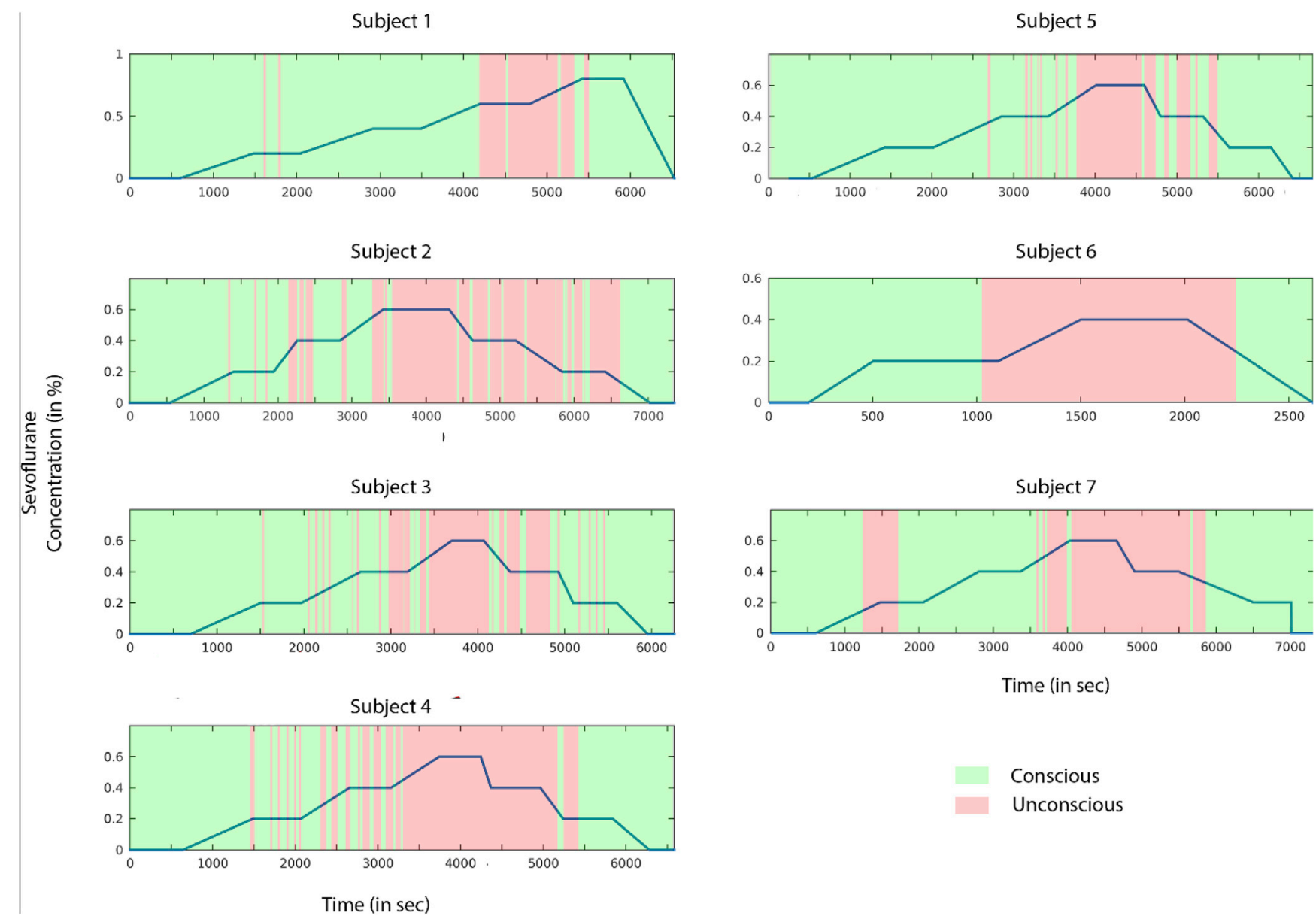

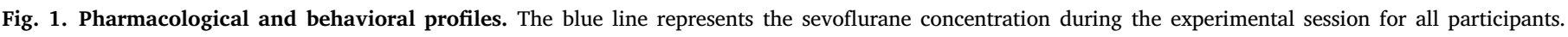
Conscious (green) and unconscious (red) segments were determined based on the responsiveness of participants. 
A

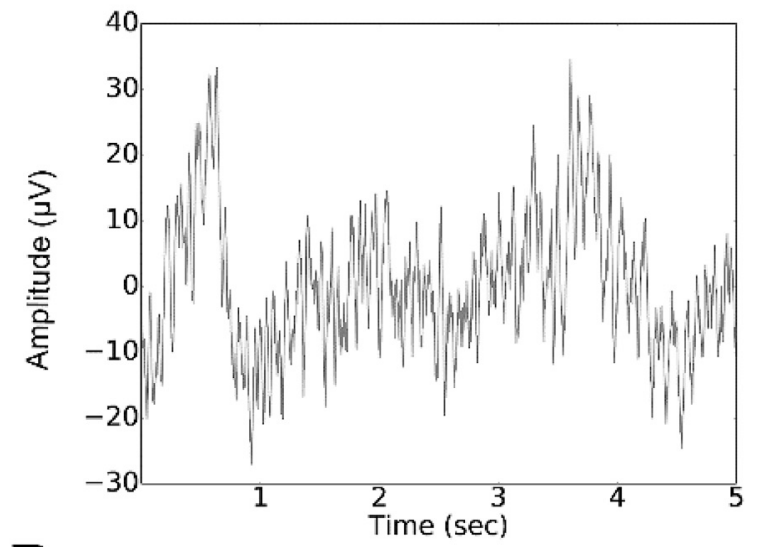

B
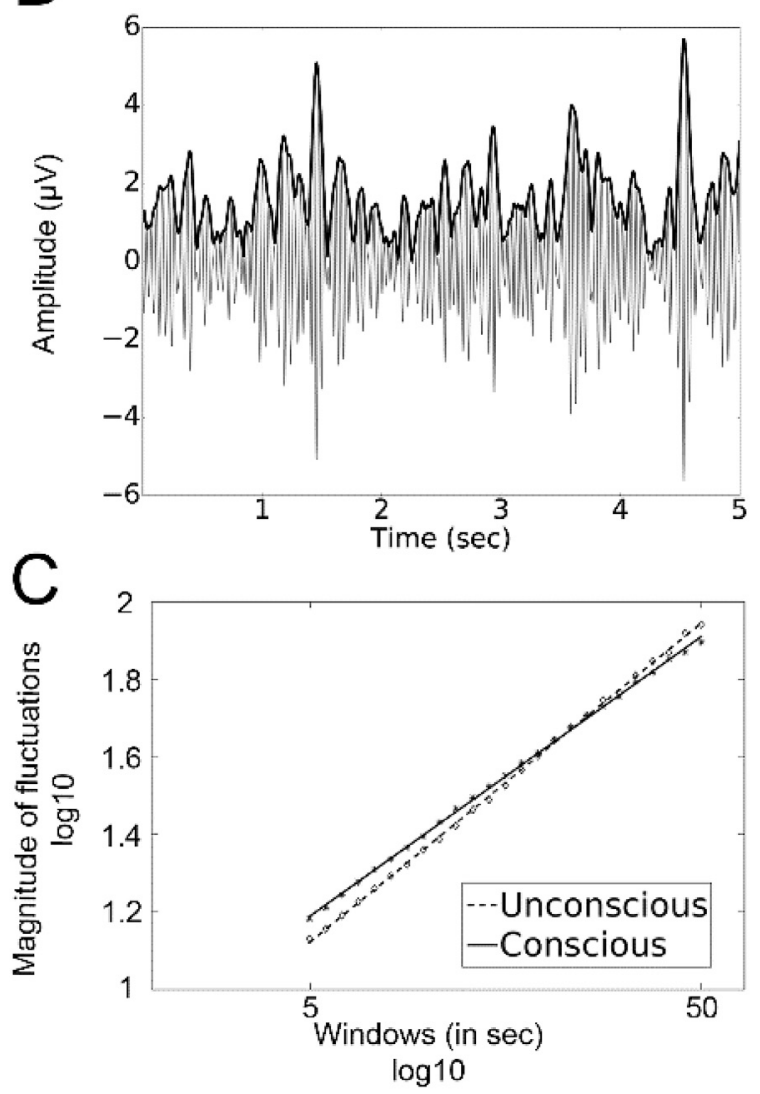

Fig. 2. Estimating scaling behavior in oscillatory amplitude using DFA. (A) A 5 s sample of raw signal from electrode Cz. (B) The filtered signal (continuous grey line) and instantaneous amplitude (bold black line) of neuronal oscillations in the beta frequency range. (C). The DFA method measures the root-meansquare fluctuation of the integrated and linearly detrended signals as a function of time-window size. The slope of the fluctuation function for consciousness (continuous line) and unconsciousness (dotted line) for all subject on electrode Cz.

permutations $=128$ ) on amplitude values and scaling exponent values. For most subjects, conscious and unconscious conditions were significantly unbalanced, resulting in a larger number of conscious samples. To address this issue, we randomly selected conscious samples in order to match the number of unconscious samples for each subject separately before performing the statistical analysis. We repeated this procedure 100 times, with different segments randomly chosen every time, and observed stable results across repetitions.
Control analysis: effect of sevoflurane concentration

To verify whether changes in oscillatory amplitude and scaling exponents can be entirely explained by changes in sevoflurane concentration rather than states of consciousness, an additional analysis was conducted. We used the sevoflurane concentration to discriminate periods of higher sevoflurane concentration $(>0.4)$ and lower sevoflurane concentration $(<0.4)$ segments in the raw EEG data across the entire recordings, during the state of consciousness only. The threshold of 0.4 was chosen by taking the median split of sevoflurane concentration ( $\min =0, \max =0.8)$. For each participant, this procedure led to two raw datasets, one for the higher sevoflurane condition and one for the lower sevoflurane condition. We computed LRTC and the instantaneous amplitude of neural oscillations using the same methods as those used for the comparison between consciousness and unconsciousness conditions. Then, we assessed statistical differences between lower and higher sevoflurane concentration at the group level using a two-sided permutationbased pseudo $t$-test corrected with maximum statistics using exhaustive permutations on amplitude values and scaling exponent values.

\section{Machine learning analysis}

In addition to investigating the contrasts between consciousness and unconsciousness using standard statistical assessments, we also assessed whether the neural markers (i.e., alpha amplitude and beta scaling exponent) that we identified as being relevant through comparisons between means also carried discriminative information at the level of single 60 -s epochs, which we also refer to as trials. To this end, we implemented a machine learning (ML) framework for trial-by-trial classification of consciousness states using spectral amplitude and scaling exponent values as features. To avoid classification bias that arises from unbalanced classes, all ML analyses were performed on balanced sets of epochs where we randomly selected conscious samples in order to match the number of unconscious samples available for each subject. This random selection was repeated 100 times and decoding performance was computed as the mean of all classification accuracies across repetitions. To avoid any bias from unbalanced classes, we used equal numbers of epochs in each class within each individual. This led to an observation space of dimension $n=476$ for the cross-subject ML framework.

Two classification procedures were explored in this study. First, the machine-learning procedure was conducted by using single feature classification in order to identify the most relevant features discriminating conscious and unconscious conditions. We then used multi-feature classification to evaluate whether the decoding accuracies could be enhanced when combining multiple features and electrodes within each classification.

Single Feature Classification: Several classification techniques were initially tested for the single feature classification procedure, including linear-discriminant analysis (LDA), k-nearest-neighbor (KNN) and support vector machine (SVM). The results were very similar across the methods, with slightly better and faster results using LDA, which was therefore chosen for this study. Within- and across-subject classification of conscious versus unconscious states were performed using LDA (Fisher, 1936). In brief, for a two-dimensional problem, the LDA algorithm tries to find a hyperplane that maximizes the mean distance between the mean of the two classes while minimizing inter-class variance.

Computation of decoding accuracy via cross-validation: At the group level, the performance of the proposed classification method was evaluated using a Leave-One-Subject-Out (LOSO) cross-validation procedure. This procedure is a special case of $k$-fold cross-validation, where all individuals except one are used for training, and the classifier is tested on the data from the omitted participant (i.e., test data). This procedure is repeated iteratively, each time leaving a different individual out of the training. The LOSO cross-validation method efficiently uses data and provides an asymptotically unbiased estimate of the averaged classification error probability over all possible training sets (Theodoridis and 
Koutroumbas, 2009). The statistical significance of the obtained decoding accuracies was evaluated by computing statistical thresholds using permutation tests $(n=1000, p<0.001)$. In other words, a null-distribution is generated by repeatedly $(n=1000)$ computing the classification accuracy obtained after randomly permuting class labels (Combrisson and Jerbi, 2015).

Multi-Feature classification: A multi-feature (MF) classification procedure was employed to determine if (and to which extent) correct classification rate can be enhanced by combining features across types (oscillation amplitude and LRTC) and/or across all electrodes. We tried several MF strategies including K-best, Forward Feature Selection, Backward Feature Elimination, and Sequential Forward Feature Selection. Optimal classification performance was obtained by using LDA. This was done within the training set, in order to conserve a strict separation between training/ hyper-parameter selection and the hold-out set. The performance of the proposed classification method was evaluated using a Leave-One-SubjectOut (LOSO) cross-validation procedure. This procedure is repeated as many times as there were participants, each time leaving a different individual out of the training data. Given that we had 7 participants; the procedure was completed in 7 iterations, with each iteration producing either a correct or an incorrect classification of the untrained, test data set. As for all other analyses, we randomly selected conscious epochs in order to match the number of unconscious samples for each subject and repeated our analyses 100 times. We then averaged the decoding accuracies across repetitions. Finally, we tested the statistical significance of all reported results using maximum statistics and derived significance thresholds using permutation testing $(\mathrm{n}=1000, \mathrm{P}<0.001)$.

\section{Results}

The scaling behavior of oscillation amplitude dynamics of conscious ( $n=477$, mean per subject $=68 \pm 21)$ and unconscious $(n=238$, mean per subject $=34 \pm 14$ ) epochs was obtained using DFA in all participants.
Fig. 1C shows an illustrative example of how scaling behavior (i.e., an estimate of the scaling exponent) was derived for beta-band amplitude dynamics at electrode $\mathrm{CZ}$ for conscious and unconscious conditions. The plot shows data for 5-50s. The linear relationship between the logarithm of the time scale and the logarithm of the amplitude fluctuations reflects power law scaling. The spatial distribution of the scaling exponents for delta, theta, alpha, beta and gamma oscillations is presented in Fig. 3. Importantly, scaling exponents values were all between 0.5 and 1 , which is consistent with the presence of LRTC in all frequency bands and for both conscious and unconscious states. This observation is in agreement with previous findings (Nikulin and Brismar, 2005; Linkenkaer-Hansen et al., 2001). Moreover, when comparing scaling exponents between conscious and unconscious epochs, significant differences were found uniquely for beta oscillation envelope ( $\mathrm{p}<0.05$, corrected). The electrodes showing this effect formed a cluster over frontocentral areas.

\section{Posterior alpha power also distinguishes between states of consciousness}

The spatial topographies of oscillation amplitude (similar to spectral power) for delta, theta, alpha, beta and gamma bands in consciousness and unconsciousness are shown in Fig. 4. The only frequency band in which we observed a significant change in mean oscillation amplitude was the alpha $(8-13 \mathrm{~Hz})$ band. Compared to consciousness, unconsciousness was associated with a significant drop in alpha amplitude over posterior brain areas $(\mathrm{p}<0.05)$. No such effects were found in the beta band where significant changes in LRTC were observed.

Importantly, we conducted an additional analysis to investigate whether changes in scaling exponents and oscillation amplitude could be driven by changes in sevoflurane concentration rather than states of consciousness. As shown in the supplementary figures, changes in sevoflurane concentration did not lead to significant changes in either scaling exponents (Fig. S1) or the instantaneous amplitude of neural oscillations (Fig. S2).

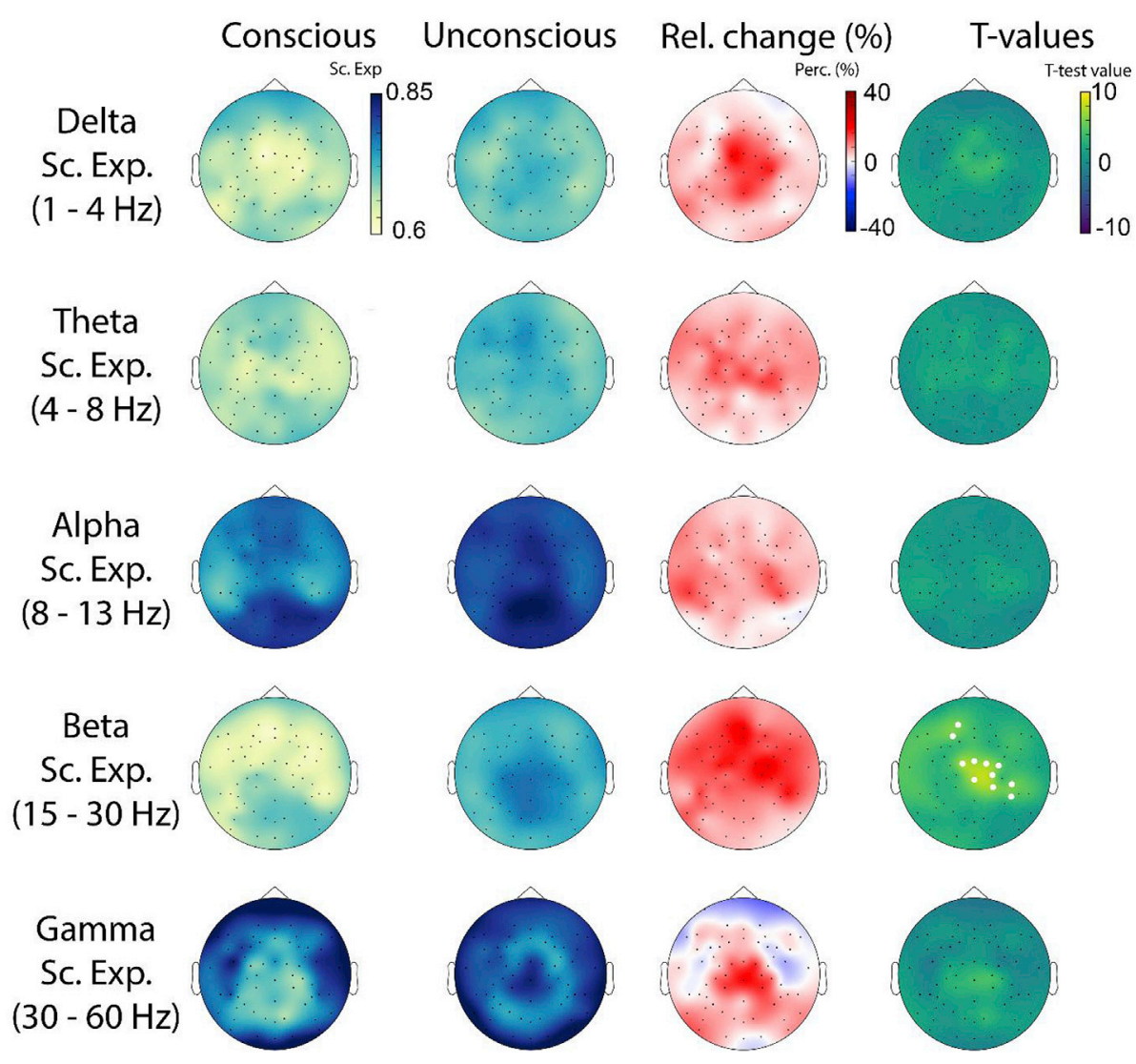

Fig. 3. Topographical plots showing the spatial distribution of mean values for delta, theta, alpha, beta and gamma scaling exponents for conscious and unconscious conditions, and the percent relative change (Relative Change $=[$ Unconsc-Consc $] /$ Consc). Topographical maps of statistical differences between consciousness and unconsciousness at the group level are also shown. White dots represent the statistical significance derived for the sample size using exhaustive permutations corrected with maximum statistics at $\mathrm{p}<0.05$. 


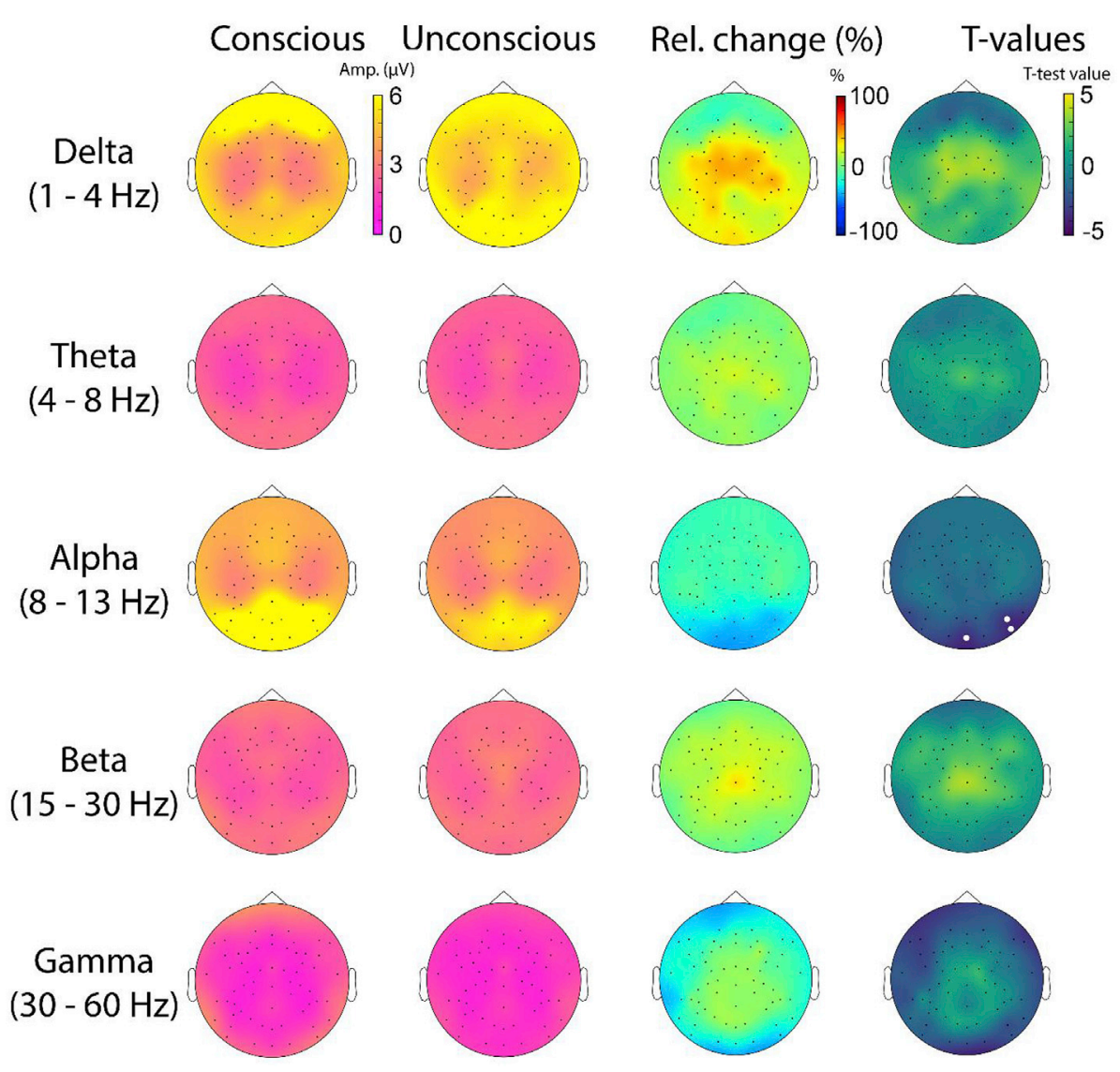

Fig. 4. Topographical plots showing the spatial distribution of mean values for delta, theta, alpha, beta and gamma amplitudes for conscious and unconscious conditions, and the percent relative change (Relative $=$ [Unconsc-Consc $] /$ Consc). Topographical maps of statistical differences between consciousness and unconsciousness at the group level are also shown. White dots represent the statistical significance derived for the sample size using exhaustive permutations corrected with maximum statistics at $\mathrm{p}<0.05$.
Classification accuracy of states of consciousness is maximal when both beta LRTC and alpha power are combined

We conducted a ML analysis to probe the robustness of the LRTC and amplitude changes across levels of consciousness. For both single feature and multi-feature classification, we restricted the choice of the classifier either to an LDA or a SVM (with a linear or RBF kernel). The LDA provided higher decoding accuracy compared to other options. As a consequence, we used an LDA algorithm in our ML pipelines. This analysis revealed that beta-band LRTC and alpha amplitude could both be used to correctly predict consciousness from unconsciousness on single trials (Fig. 5). The multi-feature analysis also gave the best results using LDA and a Sequential Forward Feature Selection. Multivariate classification using data from all electrodes yielded decoding accuracies of $75.22 \%$ for beta LRTC and $79.05 \%$ for alpha amplitude using single-trial LDA and LOSO cross-validation. Consistent with our findings in the LRTC analysis, while beta scaling exponents provided significant decoding on frontocentral channels, alpha oscillation amplitude led to significant decoding primarily over posterior regions. Interestingly, the spatial patterns of single-trial decoding accuracy (left panels in Fig. 5) closely match the topographies of differences obtained using statistical comparisons of means (Figs. 3 and 4). Moreover, performing multi-site multi-feature decoding combining the beta scaling exponents and alpha amplitudes in a common classification framework increased the overall decoding accuracy to $80.72 \%$ (Fig. 5 C). This increase, compared to the decoding accuracy obtained when using either beta LRTC or alpha amplitude individually, may indicate that LRTC and oscillation amplitude features reflect changes in distinct mechanisms underlying consciousness.

\section{Discussion}

Although spectral power has been repeatedly shown to be modulated across states of consciousness, much less is known about whether the long-range temporal properties of such oscillations vary across levels of consciousness. Given that unconsciousness is associated with a decrease in neuronal excitability and diminished information integration capacity, we hypothesized that loss of consciousness should be associated with modulations in LRTC of relevant EEG rhythms. The results reported here using anesthetic-induced unconsciousness confirm this hypothesis and provide the first evidence for an increase in LRTC in the envelope of frontocentral beta oscillations during shallow unconsciousness. Spectral power in the beta frequency range did not change. However, a significant drop in posterior alpha power was found. The diverging spatial and spectral patterns observed with LRTC and oscillation amplitude suggest that these two features reflect distinct properties of brain dynamics that may be altered during unconsciousness. Conversely, the amplitude of neuronal oscillations and their scaling behavior may underlie different mechanisms critical to maintaining consciousness.

\section{Unconsciousness is associated with increased beta LRTC}

The values of the scaling exponents of our DFA analysis are globally in agreement with values reported in previous studies and are thus consistent with the presence of LRTC in neuronal oscillations recorded with EEG/MEG (Linkenkaer-Hansen et al., 2001, 2004, Nikulin and Brismar, 2004, 2005). The increase in scaling exponents in beta-band during unconsciousness reflects greater temporal correlation in the amplitude envelope of the EEG at this frequency, and is often described as increased persistence in the signal. It has been hypothesized that this trend towards regularity may reflect a drop in neuronal excitability which may, in turn, constrain cognitive flexibility (He, 2014). The reported rise in beta-band LRTC during anesthetic-induced unresponsiveness is consistent with this explanation.

The electrode sites that showed statistically significant increases of 
A

DA (\%)

Beta Sc. Exp.

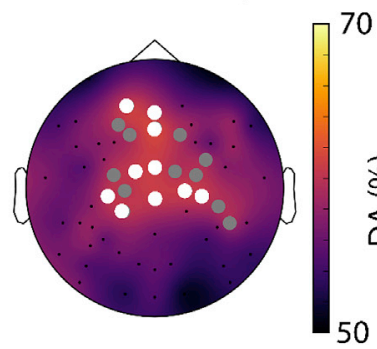

C
B

$75,22 \%$

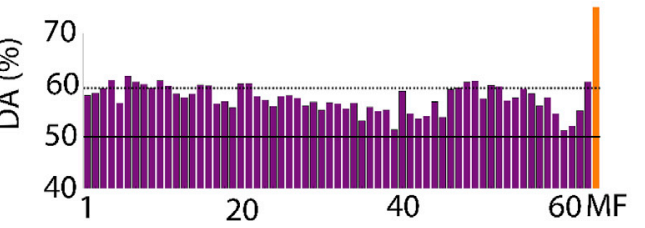

Electrodes
D

Fig. 5. Topographical maps of single-trial decoding performance (Conscious vs. Unconscious). Topographical maps (left) and bar charts (right) of the single-feature decoding accuracy (DA) for the beta band scaling exponents (A,B), alpha amplitude (C,D), as well as the beta scaling exponent and the alpha amplitude combined (E,F). All circles in panels (A), (C) and (E) indicate electrodes where the DA was statistically significant ( $p<0.001$, corrected). Among these, the ten that had the highest decoding accuracies are labeled with a white circle. The continuous horizontal lines on the bar charts depict the theoretical chance level (50\%), while the dotted lines represent the statistical significance threshold ( $p<0.001$, corrected). The orange bar to the right of each bar plot represents the decoding accuracy obtained with the multifeature decoding method described above. Alpha Amp.

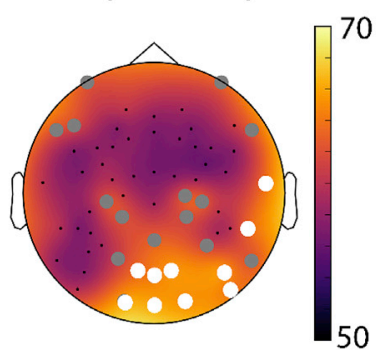

E DA (\%) Alpha Amp. + Beta Sc. Exp
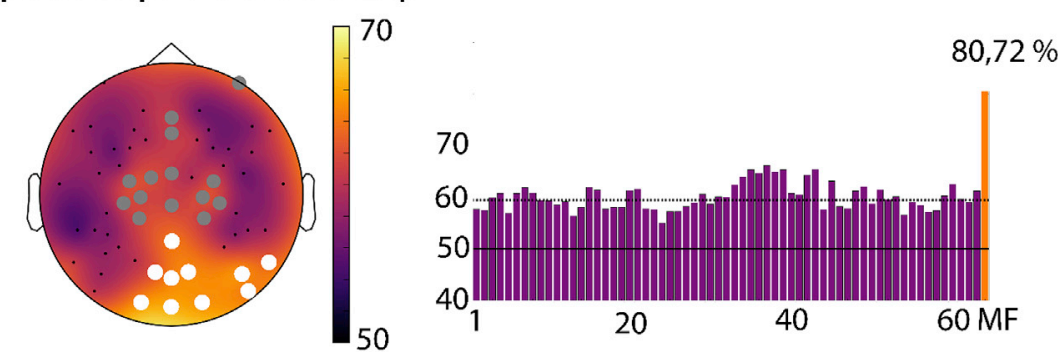

beta LRTC in the present study were broadly located over frontocentral brain regions. Incidentally, this area overlaps with the location of putative generators of sensorimotor beta oscillations (Niedermeyer and da Silva, 2005), which have been linked to signaling unchanging motor or cognitive states (Engel and Fries, 2010). The possibility that increased beta LRTC could indicate reduced switching between such states is a plausible hypothesis but it cannot be demonstrated with the currently reported data. Our assessment of changes in oscillation amplitude did not show any state-based differences in the beta band between consciousness and unconsciousness. This precludes an alternative interpretation of the results based on motor-related activations. An additional analysis conducted on the mu $(9-11 \mathrm{~Hz})$ rhythm, with the methods previously described, did not yield any significant effects in amplitude or LRTC. The fact that amplitude and LRTC differences between consciousness and unconsciousness were only visible in the beta range and not in the mu and alpha frequency bands makes an account based on motor-related activity highly unlikely.

Several studies have applied multi-scale formalisms to the raw EEG signal to monitor the depth of anesthesia (Gifani et al., 2006; Jospin et al., 2007; Lalitha and Eswaran, 2007; Nguyen-Ky et al., 2010; Liang et al., 2015). Findings from these studies suggest that the quantification of scaling exponents by applying DFA to the raw EEG signal leads to comparable, and sometimes better, classification of the depth of anesthesia compared to assessments using other known measures such as bispectral index. The objective of the present study is different. Rather than seeking to find a metric to assess the depth of general anesthesia, we set out to assess modulations in the scale-free properties of neural oscillations when participants alternate between wakefulness and sedation. By doing this we aimed to gain novel insights into the role of brain oscillations in levels of consciousness by comparing their LRTC during states of consciousness and unconsciousness. Our study is the first to use DFA specifically on band-limited oscillation amplitude signals (envelopes) in humans during wakefulness and sevoflurane-induced unconsciousness.

In a recent study, Krzemiński et al. (2017) used DFA on the amplitude of local field potentials (LFP) in monkeys and observed a decrease of LRTC during unconsciousness induced by anesthetics. The discrepancies between their results and ours could be due to a number of reasons. First, the anesthetic agents used to induce general anesthesia in monkeys differed from the one used here; the authors used propofol, ketamine, medetomidine, or ketamine and medetomidine while we used sevoflurane. Moreover, in our study the participants were purposely maintained on the border of conscious/unconscious state transitions through a sedation protocol, whereas the monkeys in the study by Krzemiński et al. (2017) underwent deep anesthesia. Lastly, the processes underlying consciousness and unconsciousness may differ between humans and primates.

To our knowledge, the present study is the first to detect a modulation in LRTC in beta oscillation amplitudes during unconsciousness. Overall, the increased beta-band LRTC we found is consistent with predictions 
based on the literature, and provides further evidence supporting the theory that anesthetics induce unconsciousness by reducing information integration and the repertoire of available cortical activation patterns (Alkire et al., 2008; Casali et al., 2013; Barttfeld et al., 2015; Hudetz et al., 2015; Solovey et al., 2015; Wang et al., 2017).

For the first time, the present study provides evidence for an increase in LRTC of beta-band oscillation amplitude during unconsciousness. This finding demonstrates that the loss of consciousness is accompanied by an augmentation of temporal persistence in neuronal oscillation amplitude, reflecting increased regularity in brain activity.

\section{Suppression of posterior alpha amplitude during anesthetic-induced unconsciousness}

Our assessment of mean oscillation amplitudes showed a significant suppression of occipital alpha, as expected from previous experimental and modeling work in anesthetic-induced unconsciousness (Gugino et al., 2001; Feshchenko et al., 2004; Blain-Moraes et al., 2015; Purdon et al., 2015; Pavone et al., 2017). Although the neurobiological basis of this occipital alpha suppression during anesthesia is not fully understood, computational models suggest that alpha suppression may be caused by alterations of hyperpolarization-activated membrane currents in the thalamus (Ying et al., 2006; Vijayan et al., 2013). Combining this occipital alpha model with a model of GABAergic inhibition enhancement involved in frontal alpha generation also accounts for alpha anteriorization (Ching et al., 2010; Purdon et al., 2013; Vijayan et al., 2013; Mukamel et al., 2014). The shallow unconsciousness induced here using low concentrations of sevoflurane only led to a drop in occipital alpha but not in alpha anteriorization, as reported previously for this data (Blain-Moraes et al., 2015) and in other analyses (Kaskinoro et al., 2015).

Supervised classification confirms distinct contributions of oscillations and LRTC

The application of a supervised learning framework allowed us to determine which features, among those discussed above, were useful for short single-trial decoding of consciousness and unconsciousness. Being able to decode conscious and unconscious states on a single-trial basis is important for clinical monitoring and the prevention of intraoperative awareness with explicit recall (Mashour et al., 2012). Our results also demonstrate that combining alpha amplitude and beta LRTC features across multiple sites yields higher decoding than using just one or the other. Furthermore, EEG channels that showed significant classification using alpha amplitudes were located over occipitoparietal cortex. This is consistent with the account of Tononi and Koch (2015), suggesting that the NCC are primarily localized to a posterior cortical hot zone that includes sensory areas. By contrast, the beta LRTC decoding results suggest the additional involvement of frontocentral areas. This finding seems to corroborate the hypothesis that a frontoparietal circuit is ignited during consciousness (Dehaene and Changeux, 2011). Further studies are needed to confirm and extend these findings. Indeed, recent results from no-report paradigms challenge the ignition of a frontoparietal network as NCC, as it may be elicited by various components such as attention, working memory or expectations (Tsuchiya et al., 2015).

\section{LRTC in anesthesia and implications for NCC}

Our findings are consistent with previous investigations of the neuronal mechanisms underlying consciousness and anesthetic-induced unconsciousness, and with existing hypotheses of the mechanisms of unconsciousness such as the cognitive unbinding theory (Mashour, 2013) and the integrated information theory (Tononi et al., 2016). Various candidates for NCC have been reported including a frontoparietal ignition network (Dehaene and Changeux, 2011) or alternately a posterior cortical hot zone (Koch et al., 2016), and often involve local and cortico-cortical oscillatory synchronization phenomena (Tononi and
Koch, 2015). The present study is the first to provide evidence for an increase in LRTC of beta-band oscillation amplitude during unconsciousness. In recent years, LRTC of EEG or MEG oscillation amplitudes has been used to investigate the neural dynamics for a number of conditions such as Alzheimer's disease, schizophrenia, and depression (Linkenkaer-Hansen et al., 2005; Montez et al., 2009; Nikulin et al., 2012). Our findings suggest that the scaling behavior of brain oscillation amplitude may constitute a distinctive feature characterizing the NCC through a characterization of the temporal properties of local and large-scale rhythmic synchronization.

\section{Limitations and future directions}

This study uses an anesthetic to induce unconsciousness, raising the possibility that the changes in beta amplitude LRTC are specific to the drug, as opposed to the state of consciousness of the participants. Our control analyses (cf. supplementary figures) demonstrate that changes in EEG oscillation amplitude and LRTC cannot be entirely explained by changes in sevoflurane concentration and thus likely reflect changes in the state of consciousness. Another valuable approach to tackle this question would be to assess changes in EEG during unconsciousness induced by molecularly distinct anesthetics or during sleep. Indeed, sleep can also be considered as a physiological state in which both the level and content of consciousness are reduced. Several studies have applied DFA to the raw EEG signal during sleep and showed increased scaling exponent during deep sleep compared to wakefulness (Lee et al., 2002; Zorick and Mandelkern, 2013; Kim et al., 2009; Lee et al., 2004). Additionally, in a recent study, the investigators applied DFA to neuronal oscillations and demonstrated that LRTCs decline as sleep deprivation progresses, and then increase during sleep (Meisel et al., 2017). By demonstrating the importance of sleep to maintain LRTCs in the human brain, the authors postulate that sleep reorganizes cortical networks towards critical dynamics for optimal functioning. Overall, these results suggest potentially similar processes between anesthetic and sleep-induced unconsciousness. However, the natural increases in LRTCs during sleep might have a functional role in bringing back cortical networks towards critical dynamics, while anesthetics might induce an abnormal increased regularity in brain signals, which may in turn reflect a drop in neuronal excitability, thus constraining cognitive flexibility.

Moreover, in our paradigm, participants were considered conscious or unconscious based on their degree of motor responsiveness to a verbal command, which is not always equivalent (Sanders et al., 2012). This study also had a small number of subjects, in part due to the demanding nature of the gradual-induction experimental paradigm. We accounted for this limitation by applying a single-epoch ML approach using all data segments aggregated across all participants $(n=476)$ while controlling for biases due to subjects' idiosyncrasies using the LOSO procedure in order to confirm and extend the observations made through standard statistical comparisons of means. Additionally, one possible opportunity for improvement would be to explore in more detail the anatomical substrates of the changes reported here. With access to individual anatomical MRI data and a larger group of participants, one could more reliably localize sources of the EEG signals and probe the scaling and power changes in cortical source space.

This study represents a first step in investigating the significance of LRTC as a potential candidate for the NCC. Indeed, DFA scaling exponents can be considered as a potential window into criticality of consciousness. The presence of LRTC has been linked to the criticality phenomenon in neuronal networks, which implies that the system is at a metastable state with a delicate balance between excitation and inhibition (Shew and Plenz, 2013). It is plausible that the increased LRTC during unconsciousness is associated with an imbalance between excitation and inhibition in neuronal networks, resulting in a loss of dynamic range (Shew et al., 2009), information transfer, and information capacity in the brain (Shew et al., 2011). Further studies are needed to assess the extent of LRTC changes across a variety of conditions involving 
alterations of consciousness and to determine the specific underlying neuronal mechanisms and functional meanings of LRTC.

\section{Conflicts of interest}

The authors declare that they do not have competing interest regarding the publication of this paper.

\section{Acknowledgments}

TT was supported by a scholarship from Fonds de Recherche Nature et Technologies (FRQNT). KJ is supported by funding from the Canada Research Chairs program and a Discovery Grant (RGPIN-2015-04854) from the Natural Sciences and Engineering Research Council of Canada, a New Investigators Award from the Fonds de Recherche du Québec Nature et Technologies (2018-NC-206005) and an IVADO-Apogée fundamental research project grant. SBM is supported by a Discovery Grant (RGPIN-2016-03817) awarded by the Natural Sciences and Engineering Research Council of Canada, and a New Investigators Award from the Fonds de Recherche du Québec - Nature et Technologies (2018NC-204435). The data acquisition was supported by the National Institutes of Health, Bethesda, Maryland, Grant R01GM098578 (to GAM) and the Department of Anesthesiology, University of Michigan.

\section{Appendix A. Supplementary data}

Supplementary data related to this article can be found at https://doi. org/10.1016/j.neuroimage.2018.05.069.

\section{References}

Akeju, O., Westover, M.B., Pavone, K.J., Sampson, A.L., Hartnack, K.E., Brown, E.N., Purdon, P.L., 2014. Effects of sevoflurane and propofol on frontal electroencephalogram power and coherence. Anesthesiol J Am Soc Anesthesiol 121, 990-998.

Alkire, M.T., Hudetz, A.G., Tononi, G., 2008. Consciousness and anesthesia. Science 322 876-880.

Bak, P., Tang, C., Wiesenfeld, K., 1987. Self-organized criticality: an explanation of the $1 / \mathrm{f}$ noise. Phys. Rev. Lett. 59, 381-384.

Barttfeld, P., Uhrig, L., Sitt, J.D., Sigman, M., Jarraya, B., Dehaene, S., 2015. Signature of consciousness in the dynamics of resting-state brain activity. Proc. Natl. Acad. Sci. Unit. States Am. 112, 887-892.

Berthouze, L., James, L.M., Farmer, S.F., 2010. Human EEG shows long-range temporal correlations of oscillation amplitude in Theta, Alpha and Beta bands across a wide age range. Clin Neurophysiol Off J Int Fed Clin Neurophysiol 121, 1187-1197.

Blain-Moraes, S., Tarnal, V., Vanini, G., Alexander, A., Rosen, D., Shortal, B., Janke, E. Mashour, G.A., 2015. Neurophysiological correlates of sevoflurane-induced unconsciousness. Anesthesiology 122, 307-316.

Bonhomme, V., Vanhaudenhuyse, A., Demertzi, A., Bruno, M.-A., Jaquet, O., Bahri, M.A., Plenevaux, A., Boly, M., Boveroux, P., Soddu, A., Brichant, J.F., Maquet, P., Laureys, S., 2016. Resting-state network-specific breakdown of functional connectivity during ketamine alteration of consciousness in volunteers. Anesthesiology 125, 873-888.

Bornas, X., Fiol-Veny, A., Balle, M., Morillas-Romero, A., Tortella-Feliu, M., 2015. Long range temporal correlations in EEG oscillations of subclinically depressed individuals: their association with brooding and suppression. Cogn Neurodyn 9, 53-62.

Boveroux, P., Vanhaudenhuyse, A., Bruno, M.-A., Noirhomme, Q., Lauwick, S., Luxen, A., Degueldre, C., Plenevaux, A., Schnakers, C., Phillips, C., Brichant, J.-F., Bonhomme, V., Maquet, P., Greicius, M.D., Laureys, S., Boly, M., 2010. Breakdown of within- and between-network resting state functional magnetic resonance imaging connectivity during propofol-induced loss of consciousness. Anesthesiology 113, 1038-1053.

Casali, A.G., Gosseries, O., Rosanova, M., Boly, M., Sarasso, S., Casali, K.R., Casarotto, S. Bruno, M.-A., Laureys, S., Tononi, G., Massimini, M., 2013. A theoretically based index of consciousness independent of sensory processing and behavior. Sci. Transl. Med. 5, 198ra105.

Ching, S., Cimenser, A., Purdon, P.L., Brown, E.N., Kopell, N.J., 2010. Thalamocortical model for a propofol-induced alpha-rhythm associated with loss of consciousness. Proc. Natl. Acad. Sci. U. S. A. 107, 22665-22670.

Cimenser, A., Purdon, P.L., Pierce, E.T., Walsh, J.L., Salazar-Gomez, A.F., Harrell, P.G., Tavares-Stoeckel, C., Habeeb, K., Brown, E.N., 2011. Tracking brain states under general anesthesia by using global coherence analysis. Proc. Natl. Acad. Sci. Unit. States Am. 108, 8832-8837.

Ciuciu, P., Varoquaux, G., Abry, P., Sadaghiani, S., Kleinschmidt, A., 2012. Scale-free and multifractal time dynamics of fMRI signals during rest and task. Front. Physiol. 3, 186.
Combrisson, E., Jerbi, K., 2015. Exceeding chance level by chance: the caveat of theoretical chance levels in brain signal classification and statistical assessment of decoding accuracy. J. Neurosci. Meth. 250, 126-136.

Dehaene, S., Changeux, J.-P., 2011. Experimental and theoretical approaches to conscious processing. Neuron 70, 200-227.

Engel, A.K., Fries, P., 2010. Beta-band oscillations-signalling the status quo? Curr. Opin. Neurobiol. 20, 156-165.

Fedele, T., Blagovechtchenski, E., Nazarova, M., Iscan, Z., Moiseeva, V., Nikulin, V.V., 2016. Long-Range Temporal Correlations in the amplitude of alpha oscillations predict and reflect strength of intracortical facilitation: combined TMS and EEG study. Neuroscience 331, 109-119.

Feshchenko, V.A., Veselis, R.A., Reinsel, R.A., 2004. Propofol-induced alpha rhythm. Neuropsychobiology 50, 257-266.

Fisher, R.A., 1936. The use of multiple measurements in taxonomic problems. Ann Eugen 7, 179-188.

Foster, B.L., He, B.J., Honey, C.J., Jerbi, K., Maier, A., Saalmann, Y.B., 2016. Spontaneous neural dynamics and multi-scale network organization. Front. Syst. Neurosci. 10 (7). https://doi.org/10.3389/fnsys.2016.00007.

Gao, J., Hu, J., Tung, W.-W., Cao, Y., Sarshar, N., Roychowdhury, V.P., 2006. Assessment of long-range correlation in time series: how to avoid pitfalls. Phys. Rev. E 73, 016117.

Gifani, P., Rabiee, H.R., Hashemi, M.R., Taslimi, P., Ghanbari, M., 2006. Nonlinear analysis of anesthesia dynamics by Fractal scaling exponent. Conf Proc Annu Int Conf IEEE Eng Med Biol Soc IEEE Eng Med Biol Soc Annu Conf 1, 6225-6228.

Gugino, L.D., Chabot, R.J., Prichep, L.S., John, E.R., Formanek, V., Aglio, L.S., 2001. Quantitative EEG changes associated with loss and return of consciousness in healthy adult volunteers anaesthetized with propofol or sevoflurane. Br. J. Anaesth. 87, $421-428$.

He, B.J., 2014. Scale-free brain activity: past, present, and future. Trends Cognit. Sci. 18, 480-487.

Hudetz, A.G., Liu, X., Pillay, S., 2015. Dynamic repertoire of intrinsic brain states is reduced in propofol-induced unconsciousness. Brain Connect. 5, 10-22.

John, E.R., Prichep, L.S., 2005. The anesthetic cascade: a theory of how anesthesia suppresses consciousness. Anesthesiology 102, 447-471.

Jordan, D., Ilg, R., Riedl, V., Schorer, A., Grimberg, S., Neufang, S., Omerovic, A., Berger, S., Untergehrer, G., Preibisch, C., Schulz, E., Schuster, T., Schröter, M. Spoormaker, V., Zimmer, C., Hemmer, B., Wohlschläger, A., Kochs, E.F., Schneider, G., 2013. Simultaneous electroencephalographic and functional magnetic resonance imaging indicate impaired cortical top-down processing in association with anesthetic-induced unconsciousness. Anesthesiology 119, 1031-1042.

Jospin, M., Caminal, P., Jensen, E.W., Litvan, H., Vallverdu, M., Struys, M.M.R.F., Vereecke, H.E.M., Kaplan, D.T., 2007. Detrended fluctuation analysis of EEG as a measure of depth of anesthesia. IEEE Trans. Biomed. Eng. 54, 840-846.

Kantelhardt, J.W., Koscielny-Bunde, E., Rego, H.H.A., Havlin, S., Bunde, A., 2001. Detecting long-range correlations with detrended fluctuation analysis. Phys Stat Mech Its Appl 295, 441-454.

Kaskinoro, K., Maksimow, A., Georgiadis, S., Långsjö, J., Scheinin, H., Karjalainen, P., Jääskeläinen, S.K., 2015. Electroencephalogram reactivity to verbal command after dexmedetomidine, propofol and sevoflurane-induced unresponsiveness. Anaesthesia 70, 190-204.

Kim, J.W., Shin, H.-B., Robinson, P.A., 2009. Quantitative study of the sleep onset period via detrended fluctuation analysis: normal vs. narcoleptic subjects. Clin. Neurophysiol. Off. J. Int. Fed. Clin. Neurophysiol 120, 1245-1251. https://doi.org/ 10.1016/j.clinph.2009.04.018.

Koch, C., Massimini, M., Boly, M., Tononi, G., 2016. Neural correlates of consciousness: progress and problems. Nat. Rev. Neurosci 17, 307-321. https://doi.org/10.1038/ nrn.2016.22.

Krzemiński, D., Kamiński, M., Marchewka, A., Bola, M., 2017. Breakdown of long-range temporal correlations in brain oscillations during general anesthesia. NeuroImage 159, 146-158.

Lalitha, V., Eswaran, C., 2007. Automated detection of anesthetic depth levels using chaotic features with artificial neural networks. J. Med. Syst. 31, 445-452.

Lee, U., Ku, S., Noh, G., Baek, S., Choi, B., Mashour, G.A., 2013. Disruption of frontalparietal communication by ketamine, propofol, and sevoflurane. Anesthesiology 118, 1264-1275.

Lee, J.-M., Kim, D.-J., Kim, I.-Y., Park, K.-S., Kim, S.I., 2002. Detrended fluctuation analysis of EEG in sleep apnea using MIT/BIH polysomnography data. Comput. Biol. Med. 32, 37-47. https://doi.org/10.1016/S0010-4825(01)00031-2.

Lee, J.-M., Kim, D.-J., Kim, I.-Y., Suk Park, K., Kim, S.I., 2004. Nonlinear-analysis of human sleep EEG using detrended fluctuation analysis. Med. Eng. Phys. 26, 773-776. https://doi.org/10.1016/j.medengphy.2004.07.002.

Lee, U., Mashour, G.A., Kim, S., Noh, G.-J., Choi, B.-M., 2009. Propofol induction reduces the capacity for neural information integration: implications for the mechanism of consciousness and general anesthesia. Conscious. Cognit. 18, 56-64.

Lee, U., Oh, G., Kim, S., Noh, G., Choi, B., Mashour, G.A., 2010. Brain networks maintain a scale-free organization across consciousness, anesthesia, and recovery: evidence for adaptive reconfiguration. Anesthesiology 113, 1081-1091.

Li, X., Wang, F., Wu, G., 2017. Monitoring depth of anesthesia using detrended fluctuation analysis based on EEG signals. J. Med. Biol. Eng. 37, 171-180.

Liang, Z., Wang, Y., Sun, X., Li, D., Voss, L.J., Sleigh, J.W., Hagihira, S., Li, X., 2015. EEG entropy measures in anesthesia. Front. Comput. Neurosci. 9. Available at: http:// www.ncbi.nlm.nih.gov/pmc/articles/PMC4332344/. (Accessed 27 February 2017).

Linkenkaer-Hansen, K., Monto, S., Rytsälä, H., Suominen, K., Isometsä, E., Kähkönen, S., 2005. Breakdown of long-range temporal correlations in theta oscillations in patients with major depressive disorder. J. Neurosci. 25, 10131-10137. 
Linkenkaer-Hansen, K., Nikouline, V.V., Palva, J.M., Ilmoniemi, R.J., 2001. Long-range temporal correlations and scaling behavior in human brain oscillations. J Neurosci Off J Soc Neurosci 21, 1370-1377.

Linkenkaer-Hansen, K., Nikulin, V.V., Palva, S., Ilmoniemi, R.J., Palva, J.M., 2004. Prestimulus oscillations enhance psychophysical performance in humans. J. Neurosci. 24, 10186-10190.

Linkenkaer-Hansen, K., Smit, D.J.A., Barkil, A., Beijsterveldt van, T.E.M., Brussaard, A.B., Boomsma, D.I., van Ooyen, A., de Geus, E.J.C., 2007. Genetic contributions to longrange temporal correlations in ongoing oscillations. J. Neurosci. 27, 13882-13889.

Lux, T., Marchesi, M., 1999. Scaling and criticality in a stochastic multi-agent model of a financial market. Nature 397, 498-500.

Mashour, G.A., 2013. Cognitive unbinding: a neuroscientific paradigm of general anesthesia and related states of unconsciousness. Neurosci. Biobehav. Rev. 37 2751-2759.

Mashour, G.A., Shanks, A., Tremper, K.K., Kheterpal, S., Turner, C.R., Ramachandran, S.K., Picton, P., Schueller, C., Morris, M., Vandervest, J.C., Lin, N., Avidan, M.S., 2012. Prevention of intraoperative awareness with explicit recall in an unselected surgical population: a randomized comparative effectiveness trial. Anesthesiology 117, 717-725.

Meisel, C., Bailey, K., Achermann, P., Plenz, D., 2017. Decline of long-range temporal correlations in the human brain during sustained wakefulness. Sci. Rep. 7. Available at: https://www.ncbi.nlm.nih.gov/pmc/articles/PMC5605531/.

Montez, T., Poil, S.-S., Jones, B.F., Manshanden, I., Verbunt, J.P.A., van Dijk, B.W., Brussaard, A.B., van Ooyen, A., Stam, C.J., Scheltens, P., Linkenkaer-Hansen, K., 2009. Altered temporal correlations in parietal alpha and prefrontal theta oscillations in early-stage Alzheimer disease. Proc. Natl. Acad. Sci. U. S. A. 106, 1614-1619.

Monto, S., Vanhatalo, S., Holmes, M.D., Palva, J.M., 2007. Epileptogenic neocortical networks are revealed by abnormal temporal dynamics in seizure-free subdural EEG. Cerebr. Cortex 17, 1386-1393. N Y N 1991.

Mukamel, E.A., Pirondini, E., Babadi, B., Wong, K.F.K., Pierce, E.T., Harrell, P.G., Walsh, J.L., Salazar-Gomez, A.F., Cash, S.S., Eskandar, E.N., Weiner, V.S., Brown, E.N., Purdon, P.L., 2014. A transition in brain state during propofol-induced unconsciousness. J. Neurosci. 34, 839-845.

Nguyen-Ky, T., Wen, P., Li, Y., 2010. An improved detrended moving-average method for monitoring the depth of anesthesia. IEEE Trans. Biomed. Eng. 57, 2369-2378.

Niedermeyer, E., da Silva, F.H.L., 2005. Electroencephalography: Basic Principles, Clinical Applications, and Related Fields. Lippincott Williams \& Wilkins.

Nikulin, V.V., Brismar, T., 2004. Long-range temporal correlations in alpha and beta oscillations: effect of arousal level and test-retest reliability. Clin Neurophysiol Off J Int Fed Clin Neurophysiol 115, 1896-1908.

Nikulin, V.V., Brismar, T., 2005. Long-range temporal correlations in electroencephalographic oscillations: relation to topography, frequency band, age and gender. Neuroscience 130, 549-558.

Nikulin, V.V., Jönsson, E.G., Brismar, T., 2012. Attenuation of long-range temporal correlations in the amplitude dynamics of alpha and beta neuronal oscillations in patients with schizophrenia. NeuroImage 61, 162-169.

Pal, D., Silverstein, B.H., Lee, H., Mashour, G.A., 2016. Neural correlates of wakefulness, sleep, and general anesthesia: an experimental study in rat. Anesthesiology 125, 929-942.

Palanca, B.J.A., Mitra, A., Larson-Prior, L., Snyder, A.Z., Avidan, M.S., Raichle, M.E., 2015. Resting-state functional magnetic resonance imaging correlates of sevofluraneinduced unconsciousness. Anesthesiology 123, 346-356.

Pavone, K.J., Su, L., Gao, L., Eromo, E., Vazquez, R., Rhee, J., Hobbs, L.E., Ibala, R., Demircioglu, G., Purdon, P.L., Brown, E.N., Akeju, O., 2017. Lack of responsiveness during the onset and offset of sevoflurane anesthesia is associated with decreased awake-alpha oscillation power. Front. Syst. Neurosci. 11. Available at: http://www. ncbi.nlm.nih.gov/pmc/articles/PMC5447687/.

Peng, C.K., Havlin, S., Stanley, H.E., Goldberger, A.L., 1995. Quantification of scaling exponents and crossover phenomena in nonstationary heartbeat time series. Chaos Woodbury N 5, 82-87.
Purdon, P.L., Pavone, K.J., Akeju, O., Smith, A.C., Sampson, A.L., Lee, J., Zhou, D.W., Solt, K., Brown, E.N., 2015. The Ageing Brain: age-dependent changes in the electroencephalogram during propofol and sevoflurane general anaesthesia. Br. J. Anaesth. 115 (Suppl 1), i46-i57.

Purdon, P.L., Pierce, E.T., Mukamel, E.A., Prerau, M.J., Walsh, J.L., Wong, K.F.K., SalazarGomez, A.F., Harrell, P.G., Sampson, A.L., Cimenser, A., Ching, S., Kopell, N.J., Tavares-Stoeckel, C., Habeeb, K., Merhar, R., Brown, E.N., 2013. Electroencephalogram signatures of loss and recovery of consciousness from propofol. Proc. Natl. Acad. Sci. U. S. A. 110, E1142-E1151.

Ranft, A., Golkowski, D., Kiel, T., Riedl, V., Kohl, P., Rohrer, G., Pientka, J., Berger, S., Thul, A., Maurer, M., Preibisch, C., Zimmer, C., Mashour, G.A., Kochs, E.F., Jordan, D., Ilg, R., 2016. Neural correlates of sevoflurane-induced unconsciousness identified by simultaneous functional magnetic resonance imaging and electroencephalography. Anesthesiology 125, 861-872.

Sanders, R.D., Tononi, G., Laureys, S., Sleigh, J.W., 2012. Unresponsiveness $\neq$ unconsciousness. Anesthesiology 116, 946-959.

Sarasso, S., Boly, M., Napolitani, M., Gosseries, O., Charland-Verville, V., Casarotto, S., Rosanova, M., Casali, A.G., Brichant, J.-F., Boveroux, P., Rex, S., Tononi, G., Laureys, S., Massimini, M., 2015. Consciousness and complexity during unresponsiveness induced by propofol, xenon, and ketamine. Curr Biol CB 25, 3099-3105.

Shew, W.L., Plenz, D., 2013. The functional benefits of criticality in the cortex. Neurosci Rev J Bringing Neurobiol Neurol Psychiatry 19, 88-100.

Shew, W.L., Yang, H., Petermann, T., Roy, R., Plenz, D., 2009. Neuronal avalanches imply maximum dynamic range in cortical networks at criticality. J. Neurosci. 29, 15595-15600.

Shew, W.L., Yang, H., Yu, S., Roy, R., Plenz, D., 2011. Information capacity and transmission are maximized in balanced cortical networks with neuronal avalanches. J Neurosci Off J Soc Neurosci 31, 55-63.

Slezin, V.B., Korsakova, E.A., Dytjatkovsky, M.A., Schultz, E.A., Arystova, T.A., Siivola, J.R., 2007. Multifractal analysis as an aid in the diagnostics of mental disorders. Nord. J. Psychiatr. 61, 339-342.

Solovey, G., Alonso, L.M., Yanagawa, T., Fujii, N., Magnasco, M.O., Cecchi, G.A., Proekt, A., 2015. Loss of consciousness is associated with stabilization of cortical activity. J Neurosci Off J Soc Neurosci 35, 10866-10877.

Suzuki, A., Katoh, T., Ikeda, K., 1998. The effect of adenosine triphosphate on sevoflurane requirements for minimum alveolar anesthetic concentration and minimum alveolar anesthetic concentration-awake. Anesth. Analg. 86, 179-183.

Theodoridis, S., Koutroumbas, K., 2009. Pattern Recognition, 4. ed. Elsevier Acad. Press, Amsterdam.

Tononi, G., Boly, M., Massimini, M., Koch, C., 2016. Integrated information theory: from consciousness to its physical substrate. Nat. Rev. Neurosci. 17, 450-461.

Tononi, G., Koch, C., 2015. Consciousness: here, there and everywhere? Phil Trans R Soc B 370, 20140167.

Tsuchiya, N., Wilke, M., Frässle, S., Lamme, V.A.F., 2015. No-report paradigms: extracting the true neural correlates of consciousness. Trends Cognit. Sci. 19, 757-770.

Vijayan, S., Ching, S., Purdon, P.L., Brown, E.N., Kopell, N.J., 2013. Thalamocortical mechanisms for the anteriorization of $\alpha$ rhythms during propofol-induced unconsciousness. J Neurosci Off J Soc Neurosci 33, 11070-11075.

Wang, J., Noh, G.-J., Choi, B.-M., Ku, S.-W., Joo, P., Jung, W.-S., Kim, S., Lee, H., 2017. Suppressed neural complexity during ketamine- and propofol-induced unconsciousness. Neurosci. Lett. 653, 320-325.

Ying, S.-W., Abbas, S.Y., Harrison, N.L., Goldstein, P.A., 2006. Propofol block of th contributes to the suppression of neuronal excitability and rhythmic burst firing in thalamocortical neurons. Eur. J. Neurosci. 23, 465-480.

Zorick, T., Mandelkern, M.A., 2013. Multifractal detrended fluctuation analysis of human EEG: preliminary investigation and comparison with the wavelet transform modulus maxima technique. PLoS ONE 8. https://doi.org/10.1371/journal.pone.0068360. 